\title{
TUBO GÁSTRICO ISOPERISTÁLTICO DE GRANDE CURVATURA GÁSTRICA COM SUTURA MECÂNICA NO TRATAMENTO CIRÚRGICO DO CÂNCER DE ESÔFAGO IRRESSECÁVEL
}

\section{Gastric tube of greater gastric curvature with stapler in surgical treatment of non resectable esophageal cancer}

\author{
José Luis Braga de AQUINO, Marcelo SAID, Luis Antonio BRANDI, \\ Jean Marc Vinagre Prado de OLIVEIRA, Diana MAZIERO, Vânia Leandro MERHI
}

\begin{abstract}
Aquino JLB, Said M, Brandi LA, Oliveira JMVP, Maziero D, Merhi VL. Tubo gástrico isoperistáltico de grande curvatura gástrica com sutura mecânica no tratamento cirúrgico do câncer de esôfago irressecável. ABCD Arq Bras Cir Dig 2009;22(3):147-52

RESUMO - Racional - Frequentemente, o diagnóstico da neoplasia do esôfago é tardio, restando como escolha terapêutica o tratamento paliativo. A transposição de tubo isoperistáltico da grande curvatura gástrica é um dos métodos de eleição que, porém, quando complicado com a deiscência da anastomose, impede a alimentação natural, comprometendo a qualidade de vida. Objetivo - Avaliar as complicações da anastomose esofagogástrica cervical com sutura mecânica com o tubo gástrico, em pacientes com câncer de esôfago irressecável. Método - Vinte e dois pacientes com câncer de esôfago irressecável foram submetidos à transposição do tubo gástrico isoperistáltico. A anastomose com o coto esofágico foi realizada com sutura mecânica circular em nível cervical. Foram avaliadas complicações sistêmicas (cardiopulmonares) e locais (a deiscência e a estenose da anastomose esofagogástrica cervical). Resultados - Dez pacientes (45,5\%) apresentaram de uma a três complicações, dos quais seis $(27,2 \%)$ com complicações sistêmicas: um (4,5\%) embolia pulmonar (evoluindo ao óbito), um infarto do miocárdio e quatro (18,2\%) com infecção de pulmão (todos com boa evolução com o tratamento clínico. Cinco pacientes tiveram complicações locais: três $(13,6 \%)$ com deiscência da anastomose e quatro $(18,2 \%)$ com estenose da anastomose, sendo que dois deles tiveram deiscência prévia. De 20 pacientes avaliados em período de seis a 18 meses, $16(80 \%)$ apresentaram deglutição satisfatória para sólidos e/ou pastosos. Conclusão - O tubo gástrico isoperistáltico de grande curvatura, com sutura mecânica, parece oferecer melhora significativa da deglutição com qualidade de vida satisfatória e morbimortalidade aceitável.

DESCRITORES - Neoplasia esofágica
\end{abstract}

\section{INTRODUÇÃO}

A neoplasia de esôfago é doença muito comum em regiões com populações de baixa renda e naquelas habituadas à ingestão de álcool e abuso de tabaco ${ }^{15,19}$. Quase sempre o diagnóstico dessa afecção é tardio, pois a disfagia - principal sintoma da neoplasia de esôfago -, ocorre somente quando mais de $2 / 3$ da luz esofágica esta preenchida por lesão tumoral. O diagnóstico retardado, perda de peso excessiva consequente à disfagia e associação de doenças cardiopulmonares decorrente do abuso de tabaco, tornam o portador dessa neoplasia paciente de difícil controle clínico, tendo o médico que o assiste poucas possibilidades terapêuticas. Este fato é muito comum, uma vez que em vários centros de tratamento da neoplasia esofágica, principalmente no mundo ocidental, apenas cerca de $20-30 \%$ dos pacientes conseguem realizar tratamento cirúrgico radical ${ }^{15,17,18}$.

Dessa forma, muitas vezes o tratamento para essa afeç̧ão torna-se paliativo, sendo que o ideal seriam

Trabalho realizado no Serviço de Cirurgia Torácica do Hospital e Maternidade Celso Pierro - PUC-Campinas, Campinas, SP, Brasil

Endereço para correspondência: José Luis de Braga Aquino, e-mail: jlaquino@sigmanet.com.br procedimentos que restaurassem satisfatoriamente a deglutição, sem a necessidade de novas internações hospitalares, com menores índices de complicações e que proporcionassem qualidade de vida mais adequada9. Assim, com o evoluir da técnica cirúrgica, passou-se procurar alternativas que atingissem essas finalidades, sendo o tubo gástrico isoperistáltico de grande curvatura um dos métodos de eleição ${ }^{18,20}$.

Entretanto, o grande óbice deste procedimento é a deiscência da anastomose do tubo gástrico com o esôfago cervical, retardando a deglutição normal e, consequentemente, comprometendo em demasia a qualidade de vida do paciente que já se apresenta com condições nutricionais bem comprometidas devido ao caráter avançado da doença $a^{7,9,18}$. Estudo anterior em 53 pacientes com câncer de esôfago irressecável no qual estes autores utilizaram tubo gástrico isoperistaltico paliativo, a anastomose esofagogástrica cervical foi confeccionada com sutura manual, com deiscência anastomótica em $29,5 \%$, o que comprometeu muito a qualidade de vida destes pacientes, impedindo que eles pudessem deglutir por via oral em média por um mês ${ }^{4}$.

Com o advento da sutura mecânica, divulgada a partir da década de 60 , tornou-se possível desenvolver nos dias atuais 
aparelhos de sutura mecânica com maior segurança, precisão e de rápido emprego, tentando assim reduzir o risco da deiscência da anastomose, além de simplificar a sua realização ${ }^{10,21,27}$. O declínio das complicações com a anastomose mecânica, ocorre devido ser esta anastomose em 2 planos, invertida, reduzindo a isquemia e necrose tecidual ${ }^{5,11}$.

Por ser o esôfago cervical o segmento que apresenta a maior incidência de deiscência anastomótica, vários autores preconizam a sutura mecânica a este nível, devida as vantagens que ela oferece $\mathrm{e}^{5,8,11,12,22,24,28}$. Nesse pressuposto, pode-se utilizar a sutura mecânica com o tubo gástrico isoperistáltico mesmo no intuito paliativo para tentar minimizar a deiscência anastomótica e oferecer qualidade de vida mais satisfatória. Além disso, com menor índice de deiscência anastomótica, diminui-se a permanência e os custos hospitalares. Há menor sofrimento dos doentes, devido às estenoses em menor número.

Assim sendo, o objetivo desse estudo é avaliar as complicações da anastomose esofagogástrica cervical com o tubo gástrico e sutura mecânica em uma série de pacientes portadores de câncer de esôfago irressecável.

\section{MÉTODOS}

No serviço de Cirurgia Tóracica do Hospital e Maternidade Celso Pierro, de agosto de 2003 a dezembro de 2008, foram avaliados 22 pacientes diagnosticados com carcinoma epidermóide irressecável de esôfago, submetidos à transposição paliativa do tubo isoperistáltico de grande curvatura por via retroesternal. Dezessete $(77,3 \%)$ eram do sexo masculino e somente cinco $(22,7 \%)$ do feminino, com idade variando de 49 a 76 anos.

\section{Avaliação pré-operatória}

O diagnóstico do câncer de esôfago irressecável foi realizado através dos seguintes exames: a) endoscopia digestiva alta, a qual evidenciou lesão tumoral no esôfago que variou de 18 até $36 \mathrm{~cm}$ da arcada dentária superior nos 22 doentes estudados tendo o exame anatomopatológico da lesão biopsiada resultado em carcinoma espinocelular; b) tomografia computadorizada de tórax evidenciando tumoração sem plano de clivagem com a aorta e/ou com a árvore traqueobrônquica em todos os pacientes; c) tomografia computadorizada de abdome não evidenciando metástase em nenhum órgão; d) broncoscopia nos que apresentavam câncer nos segmentos superior e médio do esôfago torácico, para visualizar invasão da neoplasia na árvore traqueobrônquica.

Em todos os pacientes foi realizada avaliação clínica e nutricional para avaliar se existia suporte nutricional para $\mathrm{o}$ ato cirúrgico proposto.

\section{Técnica cirúrgica}

Em todos os pacientes foi realizada a transposição de tubo gástrico isoperistático de grande curvatura gástrica. Esta técnica envolve a criação de um tubo utilizando a grande curvatura de mais ou menos $3 \mathrm{~cm}$ de largura com preservação dos vasos gastroepiplóicos direitos.

No intuito de obter este tubo, o estômago foi dividido em uma linha paralela à grande curvatura, extendendo-se do fundo ao antro gástrico, com auxílio de sutura mecânica linear cortante (Figura 1). O estômago seccionado lembra um "V", cuja parte correspondente nà pequena curvatura drena o esôfago distal em relação ao tumor (Figura 2).

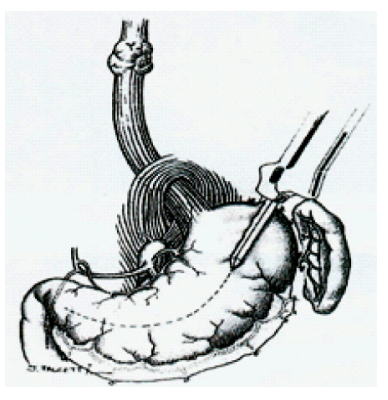

FIGURA 1 - Incisão longitudinal a aproximadamente $2 \mathrm{~cm}$ da grande curvatura

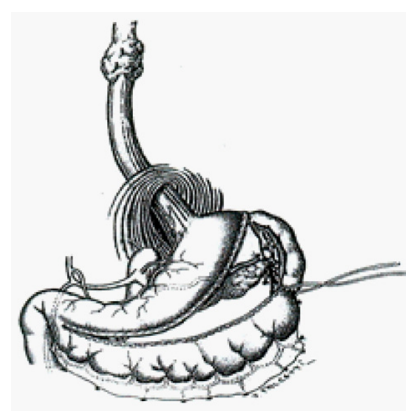

FIGURA 2 - Estômago seccionado lembrando um "V", com a preservação da artéria gastroepiplóica direita

A reconstrução do trânsito foi realizada pela transposição do tubo isoperistáltico por via retroesternal (Figuras 3 e 4), sendo realizada no pescoço a anastomose mecânica com diâmetro variável de 25 a $29 \mathrm{~mm}$. O coto do tubo gástrico por onde foi colocado o aparelho foi fechado com sutura manual. Em todos os pacientes foi realizada jejunostomia para alimentação enteral permanecendo até liberação de dieta via oral.

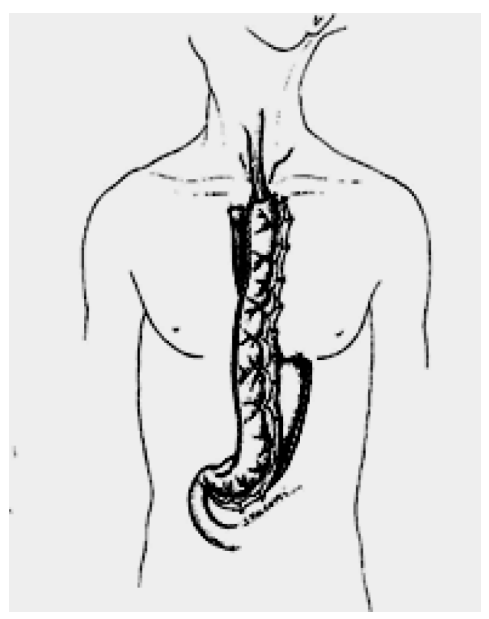

FIGURA 3 - Tubo gástrico transposto por via retroesternal, anastomosado 


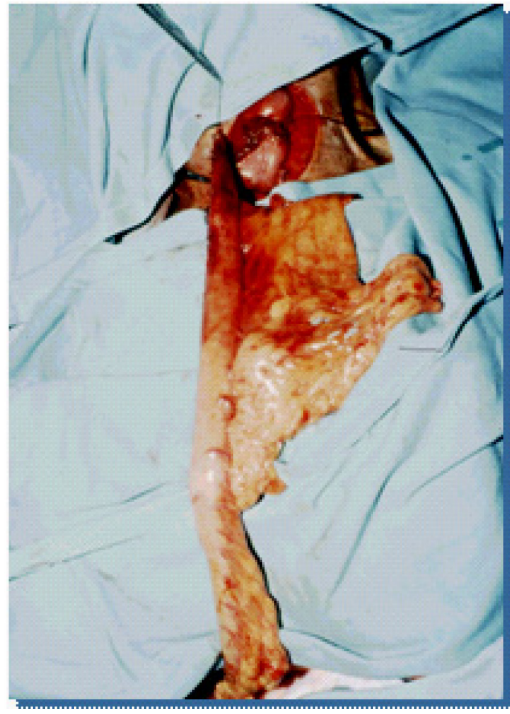

FIGURA 4 - Tubo gástrico no intra- operatório

\section{Avaliação Pós-operatória}

Foram avaliadas as complicações sistêmicas - gerais e cardiopulmonares através da avaliação clínica e métodos de imagem -, e locais - deiscências e estenoses da anastomose esofagogástrica cervical. Em relação à deiscência com sua consequente fístula, o diagnóstico era clínico, visualizandose saída de secreção gástrica ou salivar através da região cervical até o $7^{\circ}$ dia. A partir de então, não havendo sinal clínico de fistula, era realizado estudo radiográfico contrastado para avaliar extravasamento de contraste pela anastomose e se isso não ocorresse era liberada dieta oral (Figura 5).

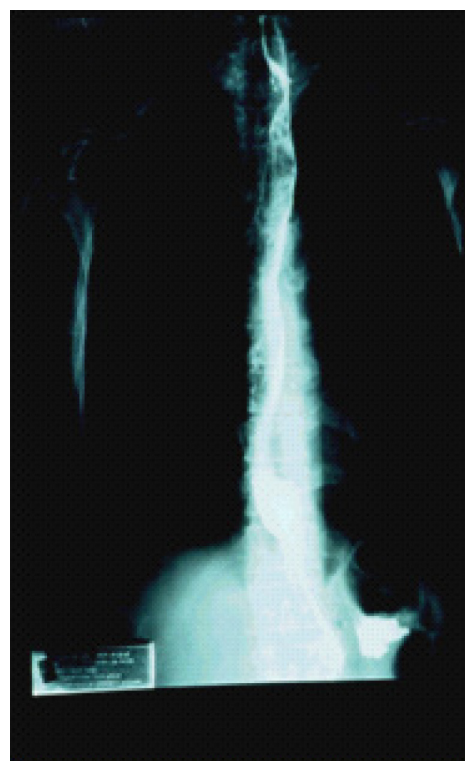

FIGURA 5 - Esofagograma após sete dias do pós-operatório

Já, em relação à estenose da anastomose, o diagnóstico era clínico através da disfagia, principalmente a partir do 30 o dia, e comprovado por estudo radiográfico contrastado e endoscópico, os quais evidenciavam a diminuição do diâmetro da anastomose cervical.

\section{RESULTADOS}

Dentre os 22 pacientes, $10(45,5 \%)$ apresentaram uma a três complicações, sistêmicas ou locais (Gráfico 1). Seis (27,2\%) evoluíram com complicações sistêmicas, sendo dois $(9,0 \%)$ com complicações cardiovasculares (um com embolia pulmonar e óbito, e o outro com infarto do miocárdio e boa evolução); e quatro $(18,2 \%)$ com infecção de pulmão, também com boa evolução clínica.

Complicações locais acometeram cinco pacientes, sendo deiscência da anastomose esofagogástrica cervical em três $(13,6 \%)$ e estenose em quatro $(18,2 \%)$, sendo dois com deiscência prévia.

Dos três pacientes que apresentaram deiscência da anastomose esofagogástrica cervical com a consequente fístula, em dois o diagnóstico foi clínico pela saída de secreção salivar e/ou gástrica pelo dreno colocado na região cervical, um deles a partir do $3^{\circ}$ e outro no $7^{\circ}$ dia, com tempo de fechamento entre o $9^{\circ}$ e $13^{\circ}$ dia do pós-operatório. Após o fechamento, foi realizado estudo radiográfico contrastado que não evidenciou nenhum extravasamento de contraste, sendo então indicada dieta oral, com progressão para sólida até a alta hospitalar entre o $14^{\circ}$ e $17^{\circ}$ dias. No paciente restante, não havendo saída de secreção salivar ou gástrica pelo dreno cervical até o $7^{\circ}$. dia, foi ele retirado e realizado estudo contrastado onde evidenciou-se pequeno extravasamento de contraste sugerindo pequena fistula na anastomose esofagogástrica cervical. Dessa forma, optou-se pelo tratamento clínico conservador com jejum e suporte nutricional através da jejunostomia até o $12^{\circ}$ dia quando, após estudo radiográfico ter mostrado cicatrização da fístula, instituiu-se dieta oral progressiva de líquida para sólida e alta hospitalar.

Nos quatro pacientes que apresentaram estenose da anastomose, o diagnóstico foi clínico pelo sintoma de disfagia progressiva entre o $25^{\circ}$ e $30^{\circ}$ dias. Em todos a diminuição da luz foi confirmada radiográfica e endoscopicamente. Neles o tratamento realizado foi dilatação endoscópica, sendo necessário 8 a 15 sessões para o retorno da ingestão normal.

Ocorreu somente um (4,5\%) óbito por embolia pulmonar.

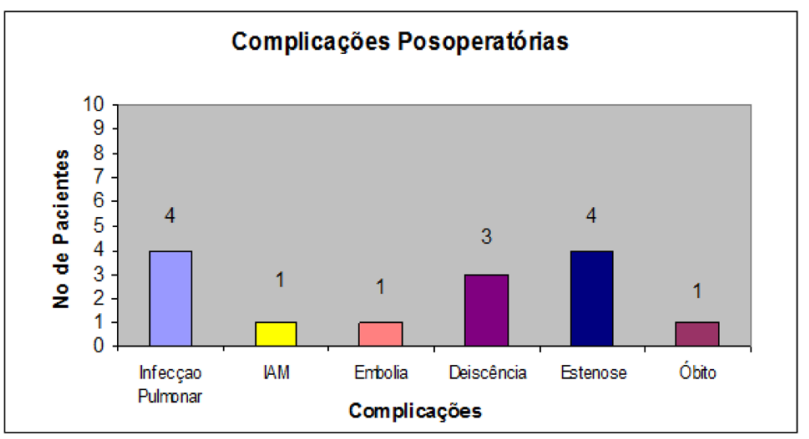

GRÁFICO 1 - Complicações pós-operatórias apresentados pelos 22 pacientes 
Em relação à satisfação com a operação, em 20 pacientes em que se conseguiu realizar acompanhamento, $16(80 \%)$ demonstraram estarem satisfeitos com o procedimento, deglutindo alimentos sólidos e/ou pastosos de maneira adequada. Alguns deles retornaram às atividades habituais. Seis pacientes moreram pela evolução natural da doença entre o $6^{\circ}$ e $11^{\circ}$ mês do pós-operatório, com média de 7,5 meses. Quatorze pacientes estão sendo acompanhados ambulatorialmente por 18 meses, com evolução satisfatória. Um paciente perdeu-se no seguimento.

\section{DISCUSSÃO}

A partir das idéias originais de Beck e Carrel $^{6}$ e os bons resultados apresentados por Postlethwait ${ }^{20} \mathrm{e}$ Speranzini et $\mathrm{al}^{23}$, passou-se a vislumbrar a possibilidade de submeter os portadores de neoplasia avançada de esôfago a procedimento cirúrgico, diferente do que se vinha propondo até então. Adotou-se o longo tubo gástrico confeccionado com a grande curvatura, isoperistáltico, anastomosado ao esôfago cervical por via retrosternal.

Este tipo de esofagoplastia obedece aos princípios básicos que orientam o cirurgião na escolha ideal de um substituto esofágico: comprimento adequado da víscera para evitar tensão, vascularização arterial e venosa satisfatória e disposição anatômica que permita boa função digestiva, em um único tempo cirúrgico ${ }^{19,29}$. A longa sutura gástrica existente neste tipo de procedimento pela utilização do grampeador linear cortante, favorece tanto o coto gástrico que ficará na cavidade abdominal quanto à sutura do tubo que será anastomosado ao esôfago cervical. Isto é realizado sem haver qualquer tipo de contaminação da ferida operatória. $\mathrm{O}$ único momento em que existe a possibilidade de contaminação é no momento da realização da anastomose entre o esôfago cervical e o tubo gástrico no pescoço.

Com a facilidade da utilização de grampeadores cirúrgicos a partir da década de 80 , a confecção do tubo gástrico de grande curvatura passou a ser um procedimento simples, rápido e seguro.

Com o intuito de preservar a maior quantidade possível de anastomose entre o território da artéria gastroepiplóica direita e da artéria gastroepiplóica esquerda na confecção do tubo, procede-se à dissecção do ligamento gastrocólico o mais próximo do cólon transverso. Este tipo de procedimento também é preconizado por Watanabe ${ }^{26}$, para evitar isquemia e desenvolvimento de fístulas pós-operatórias, principalmente quando há descontinuidade entre a arcada da artéria gastroepiplóica direita e da esquerda na grande curvatura gástrica.

A ligadura da artéria gastroepiplóica esquerda foi feita na sua emergência junto à cauda do pâncreas. Os vasos gástricos curtos foram todos ligados e a transição esofagogástrica dissecada com o intuito de realizar boa liberação do estômago. Desta maneira, conseguiu-se facilmente aplicar o grampeador linear cortante próximo do ângulo esofagogástrico, em direção paralela à grande curvatura no sentido do piloro. Obteve-se, assim, grande coto gástrico com 2 a 3 cm de diâmetro e com sua extremidade fechada e irrigada pela artéria gastroepiplóica direita, após a liberação da mesma do ligamento gastrocólico. Praticamente todo o comprimento do tubo de grande curvatura é utilizado para atingir a região cervical por via retroesternal e sem tensão, permitindo ressecção da sua porção mais cranial, que é mais sujeita à isquemia.

A anastomose esofagogástrica cervical é propensa à deiscência em uma incidência variável de 5 a $58 \%$, principalmente quando o tubo é posicionado retroesternalmente $^{1,2}$. Entretanto, a fístula consequente é controlada, na grande maioria das vezes, com o tratamento conservador, mas pode resultar em estenose no local da anastomose ${ }^{2}$.

Neste estudo, dos três pacientes $(13,6 \%)$ que apresentaram fístula da anastomose cervical em dois houve a necessidade de dilatação endoscópica pela estenose. Alguns autores referem às vezes necessidade de reoperação quando ocorre essa complicação, muitas vezes sem sucesso ${ }^{2}$. Outros autores têm demonstrado que a incidência de fistula da anastomose esofagogástrica cervical é duas vezes maior em pacientes não ressecados em relação aos ressecados, devido ao grupo não ressecado apresentar maior grau de desnutrição $0^{4,13}$.

Outros estudos tem demonstrado que além da desnutrição, a isquemia do fundo gástrico e erro da técnica cirúrgica são também fatores causais da fistula anastomótica ${ }^{3,8,25}$.

A sutura mecânica invertida é dupla e faz com que haja melhor coaptação da linha de anastomose, o que pode explicar a menor incidência de fístula com esse tipo de sutura como tem sido demonstrado por várias séries ${ }^{3,16,24}$. Em revisão recente de vários trabalhos da literatura relacionada à deiscência da anastomose esofagogástrica cervical, Chunwei $\mathrm{F}$., et $\mathrm{al}^{8}$, demonstraram que essa complicação foi significantemente maior quando foi realizada sutura manual em 263 pacientes, com 18,7\% de deiscência anastomótica, quando comparado com a sutura mecânica em 195 pacientes, com $8,2 \%$.

Em trabalho anterior, os autores deste artigo mostraram 29,2\% de deiscência da anastomose esofagogástrica cervical, com técnica manual, quando foi realizada também a transposição do tubo gástrico isoperistático com intuito paliativo ${ }^{4}$. Embora não se possa realizar comparação devido serem épocas diferentes, no estudo atual, houve praticamente duas vezes menor incidência de fístula da anastomose esofagogástrica cervical com o tubo gástrico, o que infere mais uma vez as vantagens da sutura mecânica. Daí a importância de se realizar um estudo prospectivo e comparativo para que se possa avaliar de maneira mais objetiva qual a melhor técnica de sutura a ser empregada.

Um fato bem relevante deste estudo foi estenose em outros dois pacientes mesmo não apresentando fistula cervical, a partir do $25^{\circ}$ dia de pós-operatório, o que totalizou $18,2 \%$ dessa complicação. Para alguns autores, essa complicação tem menor incidência com a sutura manual, em relação à mecânica, por ser essa invertida, dupla e além de se utilizar grampeador de menor diâmetro, devido o menor calibre do esôfago ${ }^{3,12,22}$. Nos quatro pacientes deste estudo em que houve esta complicação, a sutura mecânica foi realizada com aparelho circular de $25 \mathrm{~mm}$. Outros autores, 
entretanto, em revisão de metanálise de estudo revisado, não demonstraram diferenças significativas, independente do diâmetro da ogiva utilizada.

Uma significativa porcentagem de pacientes apresentou complicações cardiovasculares e respiratórias, aqui presente em $27,2 \%$, sendo esta similar a de outros ${ }^{2,4}$. Isso se deve, geralmente, à idade mais avançada desses pacientes, além deles apresentarem no pré-operatório alterações cardiovasculares, que necessitavam ser compensadas previamente ao ato cirúrgico. O tabagismo é outro fato relevante nesses pacientes que apresentaram essas complicações, fato também aqui presente e por outros autores ${ }^{4,8,11,16,28}$.

No que se refere à mortalidade pos-operatória imediata, ela pode variar de 0 a 42\% $\%^{2,4,14}$. Certamente, essa variação se deve às diferenças de critérios na seleção pré-operatória. Desde que se realize avaliação clínica pré-operatória bem adequada - principalmente no que se concerne as funções cardiovasculares, pulmonares e nutricionais -, a tendência é que a mortalidade seja menor. A frequência de $4,5 \%$ de óbito neste estudo é bem aceitável pelo perfil de pacientes que foram operados, além de que a causa foi por complicação cardiovascular.

A sobrevida média de 7,5 meses, apesar de ser demonstrada em apenas seis pacientes deste estudo, devido ao pequeno tempo de acompanhamento, já que os demais ainda estão sendo avaliados, também é similar à série de outros que utilizam esse tipo de procedimento ${ }^{2,4}$. Esse tempo é aceitável em vista do caráter paliativo do tratamento e da doença avançada vigente.

Tem havido na literatura relatos quanto à maior sobrevida de pacientes portadores de neoplasia avançada do esôfago, à medida que se consegue paliação da disfagia, da maneira mais adequada possível. Os métodos cirúrgicos são os que melhor restauram a qualidade da deglutição, por proporcionar melhora nutricional, diminuição da possibilidade de aspiração, broncopneumonia, beneficio emocional e psicológico, proporcionado pela nova capacidade de deglutição adequada ${ }^{4,13,14}$.

\section{CONCLUSÃO}

O tubo gástrico isoperistático da grande curvatura com sutura mecânica, parece oferecer melhora significativa da deglutição com qualidade de vida satisfatória e morbimortalidade aceitável, em vista do perfil dos pacientes em que se atua. Pode ser utilizado no tratamento cirúrgico paliativo das neoplasias malignas do esôfago.

Aquino JLB, Said M, Brandi LA, Oliveira JMVP, Maziero D, Merhi VL. Gastric tube of greater gastric curvature with stapler in surgical treatment of non resectable esophageal cancer. ABCD Arq Bras Cir Dig 2009;22(3):147-52

ABSTRACT-Background - The diagnose of esophageal cancer is in general done clinically late, remaining the palliative treatment the only possibility to improve the quality of life. The isoperistaltic gastric tube transposition may be used, however, if leakage happens, the swallow may be hindered, compromising deglutition. Aim - To evaluate the complications that can happen in the cervical esophagogastric anastomosis done with stapler, in patients with non resectable esophageal cancer. Method - Twenty two patients with non resectable esophageal cancer were submitted to an isoperistaltic gastric tube transposition. The esophagogastric anastomosis was made with circular stapler. Systemic and local complications were evaluated. Results - Ten patients (45,5\%) presented 1 to 3 complications, and in $6(27,2 \%)$ of them, systemic ones. There were one (4,5\%) case of lung embolism (with death), one miocardial infarction and four lung infections (all of them with good clinical evolution). Five had local complications; in three $(13,6 \%)$, anastomotic leakage, and in four $(18,2 \%)$, anastomotic stricture among the ones that had previous leakage. Twenty patients were followed through 11 months, and $16(80 \%)$ of them maintained satisfactory swallow to solid and/or semi-solid meals. Conclusion The isoperistaltic gastric tube of greater curvature with stapler suture seems to offer significant improvement on swallow with satisfactory quality of life and acceptable morbi-mortality.

HEADINGS- Esophageal carcinoma

\section{REFERÊNCIAS}

1. Abe S, Tachibana M, Shimokawa T, et al.- Surgical treatment of advanced carcinoma of the thoracic esophagus. Surg Gynocol Obstet, 1993, 177(4): 115-120.

2. Alcantara PS, Spencer-Netto FA, Silva-Junior JF, et al.- Gastro-esophageal isoperistaltic bypass in the palliation of irresectable thoracic esophageal cancer. Int Surg, 1997, 82(3): 249-253.

3. Aquino JLB, Camargo JGT, Said MM, et al.- Avaliação da anastomose esofagogástrica cervical com sutura mecânica e manual em pacientes com megaesôfago avançado. Rev. Col. Bras. Cir., 2009, 36(1): 19-23.

4. Aquino JLB, Muraro CLPM, Camargo JGT, et al.- Derivação retroesternal com o tubo gástrico isoperistáltico no carcinoma irressecável de esôfago. Rev. Brás. Cir., 2003; 30(2): 142-147.

5. Aquino JLB.- Avaliação da anastomose esofagogástrica cervical com sutura mecanica em pacientes com megaesôfago avançado. Rev. Col. Brás. Cir., 2005; 3:143-146.

6. Beck C, Carrell A.- Demonstration of specimens illustrating a method of formaition of a prethoracic esophagus. Illinois Med J, 1905, 7: 463-468.

7. Beitler AL, Urschel JD.- Comparison of Stapled and Hand-sewn Esophagogastric Anastomoses. Am J Surg. 1998; 175: 337-40.
8. Chunwei F, Qingzeng N, Jianliang L, et al.- Cervical Esophagogastric Anastomosis with a New Stapler in the Surgery of Esophageal Carcinoma. Eur J Cardiothorac Surg. 2005 Aug; 28(2): 291-5.

9. Gurski RR, SchiirmerCC, Tonedo JE Jr, et al.- Delay Cervical Anastomosis of the Esophagus for Esophageal Carcinoma. Int Surg. 1997 Jan-Mar;82(1):56-9.

10. Hopkins RA, Alexander JC, Postlethwait RW.- Stapled esophagogastric anastomosis. Am. J. Surg., 1984; 147: 237-238.

11. Hsu HH, Chen JS, Hsuang PM, et al.- Comparison of Manual and Mechanical cervical esophagogastric Anastomosis after Esopahgeal Resection for Esquamous Cell Carcinom: a Prospective Randomized Controlled Trial. Eur Cardiothoracic Surg, 2004, Jun; 25(6): 1097-101.

12. Law S, Fok M, Chu KM, et al.- Comparison of Hand-sewn and Stapled Esopahogastric Anastomosis After Esophageal Resection for Cancer: a Prospective Randomized Controlled Trial. Annals of Surgery. 1997, Aug, 226(2): 169-173.

13. Lorentz T, Folk M, Wong J.- Anastomotic leakage after resection and bypass for esophageal cancer: lesson learned from the past. World J Surg, 1989, 13(4): 472477. 
14. Meunier B, Spiliopoulos Y, Stasik C, et al.- Retroesternal Bypass Operation for Unresectable Squamous Cell Cancer of the Esophagus. Ann Thorac Surg, 1996 Aug;62(2):373-7.

15. Orel JJ, Vidmar SS, Hrabar BA.- Intrathoracic gastric and jejunal bypass for palliation of nonresectable esophageal carcinoma. Int Surg, 1982, 67(2): 147-151.

16. Orringer MB, Marshall B, Iannettoni.- Eliminating the Cervical Esophagogastric Anastomotic Leak With a Side-to-Side Stapled Anastomosis. J Thorac Cardiovasc Surg, 2000;119:277-288.

17. Orringer MB, Marshall B, Stirling MC.- Transhiatal esophagectomy for benign and malignant disease. Thorac. Cardiovasc. Surg., 1993; 105: 265-276.

18. Orringer MB, Sloan H.- Substernal Gastric Bypass of the Excluded Thoracic Esophagus for Palliation of Esophageal Carcinoma. J. Thorac Cardiovasc Surg. 1975, Nov;70(5): 836-51.

19. Pinotti, HW, Felix VN, Raia AA.- Revisão e análise crítica das técnicas de restabelecimento do trânsito esofágico. Anais Paul Med Cir, 1978, 105: 1-28

20. Postlethwait RW.- Technique for isoperistaltic gastric tube for esophageal bypass. Ann Surg., 1979, 189(6): 673-676.

21. Ravitch MM, Steichen FM.- A stapling instrumental for end-to-end inverting anastomosis in the gastrointestinal tract. Ann. Surg., 1979; 189: 791-797.
22. Santos RS, Raftopoulos Y, Singh D, et al-. Utility of Total Mechanical Stapled Cervical Esophagogastric Anastomosis After Esophagectomy: a Comparison to Convetional Anastomotic Technique. Surgery. 2004 Oct; 136(4): 917-25.

23. Speranzini MB, Fujimura I, Pires PW.- Esofagoplastia com tubo gástrico isoperistátilco em derivação no tratamento do câncer de esôfago torácico: estudo de 13 casos. Rev Assoc. Med Brás, 1989, 35(3):91-98.

24. Urschel JD, Blewett CJ, Bennet WF, et al.- Handsewn or Stapled Esophagogastric Anastomoses After Esophagectomy for Cancer: Meta-analysis of Randomized Controlled Trials. Dis Esophagus. 2001;14(3-4): 212-7.

25. Urschel JD.- Esophagogastrostomy asnastomotic leaks complicating esophagectomy: a review. Am J Surg 1995; 169: 634-640.

26. Watanabe H.- The techniques to protect the anastomotic leakage of esophagogastrostomy at neck. Nippon Geka Gakkai Zasshi, 1996, 97(6):432-436.

27. Wong J, Cheund H, Lui R, Fan YW, et al.- Esophagogastric anastomosis performed with a stapler: the occurrence of leakage and stricture, Surgery, 1987; 101: 408-415.

28. Zhou NK, Liu X, Liu X, Tian XD, et al.- The Application of Stapling Device in Surgery of Carcinoma of the Esophagus and Cardia. Zhonghua Yi Xue Za Zhi.2006, Jun, 6;86(21): 1450-2.

29. Zilberstein B, Cecconello I, Pollara WM.- Emprego do jejuno no restabelecimento do trânsito esofágico, análise crítica das técnicas e dos resultados. In Pinotti HWCondutas técnicas na cirurgia do esôfago. Cidade. Kronos, 1982, pp.80.

Fonte de financiamento: não há Conflito de interesse: não há Recebido para publicação: 04/02/2009 Aceito para publicação: 09/04/2009 